\title{
Improving chemotherapy outcome in early breast cancer
}

\author{
Anurag Gupta ${ }^{1}$, Rohit Joshi \\ ${ }^{1}$ Department of Breast \& Endocrine Surgery, Lyell McEwin and Modbury Hospitals, Northern Adelaide Local Health Network, South Australia, \\ Australia; ${ }^{2}$ Department of Medical Oncology, Lyell McEwin Hospital, University of Adelaide, Adelaide, South Australia, Australia \\ Correspondence to: Mr. Anurag Gupta, MS, FRACS. Department of Breast \& Endocrine Surgery, Lyell McEwin Hospital, Haydown Road, Elizabeth \\ Vale, SA 5112, Australia. Email: anurag.gupta@sa.gov.au. \\ Provenance: This is an invited article commissioned by the Section Editor Xiao-Li Liu (Deputy Director of Science and Education Department, \\ Department of Thyroid Surgery, China-Japan Union Hospital of Jilin University, Changchun, China). \\ Comment on: Early Breast Cancer Trialists' Collaborative Group (EBCTCG). Increasing the dose intensity of chemotherapy by more frequent \\ administration or sequential scheduling: a patient-level meta-analysis of 37298 women with early breast cancer in 26 randomised trials. Lancet \\ 2019;393:1440-52.
}

Submitted Jun 30, 2019. Accepted for publication Aug 12, 2019.

doi: $10.21037 /$ gs.2019.08.04

View this article at: http://dx.doi.org/10.21037/gs.2019.08.04

The growing understanding of early breast cancer as a systemic disease has firmly established the role of chemotherapy. The addition and modification of chemotherapy to standard breast cancer treatment has substantially improved outcomes in the early breast cancer patients (1).

Early Breast Cancer Trialists' Collaborative Group (EBCTCG) was established in 1985 to coordinate individual-patient-level meta-analysis of all randomised trials of adjuvant treatments (2). A meta-analysis in 2012 from this group, included 123 randomised trials, revealed that standard combination chemotherapy that included anthracyclines and taxanes, reduces breast cancer mortality in women with early breast cancer, by about a third when compared to no chemotherapy (3). It is noted that absolute benefit from chemotherapy for a woman with early breast cancer depends on her absolute risk of recurrence and death. The proportional reduction of recurrence and mortality is independent of biological characteristics of tumour.

Despite ongoing research looking into newer and improved chemotherapeutic agents and their combinations, current standard combination of anthracyclines and taxanes provide the best outcome to date. Several trials comparing doses of anthracyclines have shown no significant benefit from dose-escalation beyond standard doses (4), however lesser benefit was noted when doses were used less than current standard regimen (5). This suggests that increasing dose alone does not improve the outcome. Anthracycline based chemotherapy is dose dependent from the overall perspective of cardiac toxicity. The risk versus benefit ratio of giving more than 6 infusions of anthracyclines is poor. Most of the modern chemotherapy regimens would add a taxane to anthracycline chemotherapy for the best possible benefit.

Even with well-established superiority of anthracyclines and taxane combination, optimal timing and dosing of this regimen has been a matter of discussion for a long time. Basic cellular and cytokinetic modelling has shown that increasing dose intensity could improve tumour cell kill, decreasing regrowth of tumour cells between chemotherapy cycles therefore improving likelihood for cure $(6,7)$.

This EBCTCG meta-analysis is another important step in exploring further improvement in chemotherapy delivery (8). This patient-level meta-analysis looked at 26 randomised trials (involving 37,298 women) investigating two different ways of increasing chemotherapy intensity but not increasing total dose. Firstly, by shortening the time between chemotherapy cycles (dose dense chemotherapy) or secondly by administering individual drug sequentially (sequential scheduling). This meta-analysis compared 2-weekly dose dense chemotherapy with 3-or 4-weekly cycles, and trials of sequential versus concurrent anthracycline and taxane administration.

The study shows that by increasing the dose intensity of the standard chemotherapy regimens (based on 
anthracycline and taxane) for early breast cancer by either of the two ways (allowing full dose treatment with each agent), reduces 10-year recurrence risk and breast cancer mortality by an additional $10-15 \%$. This is already on top of the known one third reduction of breast cancer death with the use of standard chemotherapy. Therefore, this additional benefit would essentially mean an overall reduction of around $40 \%$ from breast cancer related death in first 10 years, with dose-intense adjuvant chemotherapy, when compared to no chemotherapy in early breast cancer patients.

This meta-analysis (8) noted that despite significant variability in study designs, chemotherapy combinations, doses and scheduling, the absolute risk reduction was consistently similar in trials comparing 2 -weekly versus 3 -weekly chemotherapy cycles and in those sequential versus concurrent scheduling. However, the risk reduction was noted to be smaller in trials where less than six cycles of dose-dense chemotherapy were given.

Increased efficacy of taxane administration with increased dose-intensity, regardless of the agent used or frequency of administration, is another important finding of this study. None of the 26 trials included in this metaanalysis had taxane dose intensification without addition of anthracyclines.

This EBCTCG meta-analysis showed reduction in contra-lateral new primary breast cancer risk in $0-4$ years following dose intense chemotherapy, but not thereafter. The authors believed this could be explained by chemotherapy eradicating or delaying the development of subclinical contra-lateral breast cancer but having minimal effect on future contra-lateral disease (8). In other words, this effect is likely due to dose intense chemotherapy killing the clones of early cancer cells in the contra-lateral breast, thus reducing the incidence of breast cancer in the initial few years. As the newer clones of cancer cells are being generated after the first 4 years, this reduction is not seen thereafter.

There was no specific patient or tumour characteristics that predicted higher or lesser benefit from dose intense chemotherapy. Proportional benefit is noted to be independent of hormone receptor, nodal status or other tumour characteristics, therefore dose intense chemotherapy is beneficial to most women with early breast cancer who are offered chemotherapy.

There were no unexpected toxicity signals or increased risk of death with cytotoxic dose intensification. Despite having limited information about exact causes of death amongst patients, there was no obvious increased cardiovascular or haematological mortality in patients receiving dose-intense chemotherapy. Based on patient reported outcome trials it was noted that quality of life was worse during dose-intense chemotherapy, when compared with standard regimen. However, it returned to similar QOL, once treatment is ended.

Even though as current standard clinical practice, women with larger tumours, malignant lymph node(s), grade 3 tumours, ER negative, Her2 positive, lymphovascular invasion, high $\mathrm{Ki}-67$ or $\mathrm{MiB}$ score, younger age, are the classic patient groups who are offered chemotherapy. There are other subtypes, which may increase the risk of cancer recurrence and also warrant discussion regarding adjuvant chemotherapy.

However, the bigger question-'which women with early breast cancer should truly be offered which type of chemotherapy' still remains a work in progress. This metaanalysis provides important finding of additional benefit with dose intense chemotherapy to women who are offered chemotherapy. The risk versus benefit ratio seems to favour dose-dense chemotherapy.

\section{Acknowledgments}

None.

\section{Footnote}

Conflicts of Interest: The authors have no conflicts of interest to declare.

Ethical Statement: The authors are accountable for all aspects of the work in ensuring that questions related to the accuracy or integrity of any part of the work are appropriately investigated and resolved.

\section{References}

1. Cossetti RJ, Tyldesley SK, Speers CH, et al. Comparison of breast cancer recurrence and outcome patterns between patients treated from 1986 to 1992 and from 2004 to 2008. J Clin Oncol 2015;33:65-73.

2. Early Breast Cancer Trialists' Collaborative Group (EBCTCG). Increasing the dose intensity of chemotherapy by more frequent administration or sequential scheduling: a patient-level meta-analysis of 37298 women with early breast cancer in 26 randomised trials. Lancet 
2019;393:1440-52.

3. Early Breast Cancer Trialists' Collaborative Group (EBCTCG), Peto R, Davies C, et al. Comparisons between different polychemotherapy regimens for early breast cancer: meta-analyses of long-term outcome among 100,000 women in 123 randomised trials. Lancet 2012;379:432-44.

4. Henderson IC, Berry DA, Demetri GD, et al. Improved outcomes from adding sequential Paclitaxel but not from escalating Doxorubicin dose in an adjuvant chemotherapy regimen for patients with node-positive primary breast cancer. J Clin Oncol 2003;21:976-83.

5. Budman DR, Berry DA, Cirrincione CT, et al. Dose and

Cite this article as: Gupta A, Joshi R. Improving chemotherapy outcome in early breast cancer. Gland Surg 2019;8(5):585-587. doi: $10.21037 /$ gs.2019.08.04 dose intensity as determinants of outcome in the adjuvant treatment of breast cancer. The Cancer and Leukemia Group B. J Natl Cancer Inst 1998;90:1205-11.

6. Norton L. A Gompertzian model of human breast cancer growth. Cancer Res 1988;48:7067-71.

7. Norton L. Evolving concepts in the systemic drug therapy of breast cancer. Semin Oncol 1997;24:S10-3-S10-10.

8. Early Breast Cancer Trialists' Collaborative Group (EBCTCG). Increasing the dose intensity of chemotherapy by more frequent administration or sequential scheduling: a patient-level meta-analysis of 37298 women with early breast cancer in 26 randomised trials. Lancet 2019;393:1440-1452. 